$\square$ Math-Net.Ru

Общероссийский математический портал 
Ю. В. Покорный, Т. В. Белоглазова, Е. В. Дикарева, Т. В. Перловская, О функции Грина для локально взаимодействующей системы обыкновенных уравнений разного порядка, Матем. заметки, 2003, том 74, выпуск 1, 146-149

DOI: https://doi.org/10.4213/mzm588

Использование Общероссийского математического портала Math-Net.Ru подразумевает, что вы прочитали и согласны с пользовательским соглашением 
http://www. mathnet.ru/rus/agreement

Параметры загрузки:

IP: 3.82 .47 .9

26 апреля 2023 г., 05:16:14

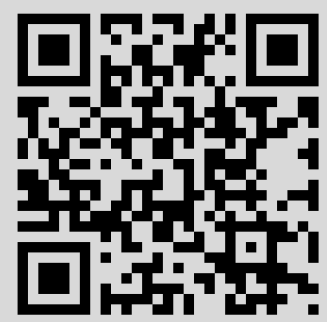




\section{О ФУНКЦИИ ГРИНА ДЛЯ ЛОКАЛЬНО ВЗАИМОДЕЙСТВУЮЩЕЙ СИСТЕМЫ ОБЫКНОВЕННЫХ УРАВНЕНИЙ РАЗНОГО ПОРЯДКА}

\section{Ю. В. Покорный, Т. В. Белоглазова, Е. В. Дикарева, Т. В. Перловская}

На промежутке $[0, l]$ рассматриваются дифференциальные уравнения

$$
\begin{aligned}
\left(p_{1} u_{1}^{\prime \prime}\right)^{\prime \prime}=f_{1}(x), & x \neq \xi, \\
-\left(p_{2} u_{2}^{\prime}\right)^{\prime}=f_{2}(x), & x \neq \xi .
\end{aligned}
$$

Первое из них возникает при описании поперечных деформаций классического стержня, а второе обычной струны (или продольных деформаций стержня). В точке $\xi$ (где, естественно, $0<\xi<l$ )

Работа выполнена при поддержке Российского фонда фундаментальных исследований, грант № 01-01-00418, Минобразования РФ (КЦ СПбГУ), грант № Е00-1.0-154, и программы “Университеты России", проект УР.04.01.047.

(C) Ю. В. ПОКОРНЫЙ, Т. В. БЕЛОГЛАЗОВА, Е. В. ДИКАРЕВА, Т. В. ПЕРЛОВСКАЯ

2003 
оба уравнения выключаются, так что фактически (1), (2) - это система четырех уравнений. Однако нас интересуют лиш решения, непрерьвно склеенные в точке $x=\xi$, что значит $u_{1}(\xi-0)=u_{1}(\xi+$ $0), u_{2}(\xi-0)=u_{2}(\xi+0)$. Более того, в этой точке непрерьвно склеены и решения разных уравнений, т.е.

$$
u_{1}(\xi \pm 0)=u_{2}(\xi \pm 0) .
$$

В этой же точке мы предполагаем выполненным условие взаимодействия (трансмиссии)

$$
\Delta\left(p_{1} u_{1}^{\prime \prime}\right)^{\prime}(\xi)+\Delta\left(p_{2} u_{2}^{\prime}\right)(\xi)=0,
$$

где через $\Delta \varphi(\xi)$ обозначается скачок $\varphi$ в точке $\xi$, т.е. $\Delta \varphi(\xi) \doteq \varphi(\xi+0)-\varphi(\xi-0)$. Допуская у $u_{1}(x)$ потерю гладкости в точке $\xi$, мы предполагаем при этом

$$
\left(p_{1} u_{1}^{\prime \prime}\right)(\xi-0)=\left(p_{1} u_{1}^{\prime \prime}\right)(\xi+0)=0 .
$$

Последнее при разговоре о стержне соответствует тому, что в точке $x=\xi$ его излома оба его куска шарнирно скреплены (склепаны). Если на концах $x=0, x=l$ отрезка поставить стандартные условия закрепления, т.е.

$$
u_{1}(0)=u_{1}^{\prime}(0)=0, \quad u_{1}(l)=u_{1}^{\prime}(l)=0, \quad u_{2}(0)=u_{2}(l)=0,
$$

то мы можем смотреть в целом на систему (1)-(6) как на краевую задачу, моделирующую, например, деформации небольшого канатного моста. При $f_{2} \equiv 0$ второе уравнение (2) вместе с условиями $(3),(4)$ заменяется, как несложно проверить, условием

$$
\Delta\left(p_{1} u_{1}^{\prime \prime}\right)^{\prime}(\xi)+\gamma u_{1}(\xi)=0, \quad \gamma>0,
$$

что вместе с (5) приводит (1) к модели двухзвенной цепочки стержней с упругой опорой в месте стыка $(x=\xi)$. Подобные нестандартные задачи для одного скалярного уравнения изучались ранее в [1]. В нашей ситуации можно говорить о задаче награфе типа креста, на двух ребрах которого задано уравнение типа (1), а на двух остальных - типа (2). Однако внешне физическое описание более естественно в терминах не четырех, а двух функций, причем заданных на общей области определения.

Можно считать, что $f_{1}$ и $f_{2}$ есть обобщенные производные $\left(f_{1}=F_{1}^{\prime}, f_{2}=F_{2}^{\prime}\right)$ от функций ограниченной вариации. Физичность условий требует предположения $f_{1}(\xi)=f_{2}(\xi)$, что в случае скачков $F_{1}$ и $F_{2}$ в точке $\xi$ означает совпадение атомов меры или - физически - общую для обеих функций $u_{1}, u_{2}$ сосредоточенную силу.

1. Задача (1)-(6) рассматривается в классе достаточно гладких (при $x \neq \xi$ ) функций $u(x)=$ $\left\{u_{1}(x), u_{2}(x)\right\}$ на $[0, l]$. Далее во всех формулировках и условиях мы предполагаем, что $x \neq \xi$ без дополнительных оговорок.

Всюду далее считаем, что $p_{1}(\cdot)$ и $p_{2}(\cdot)$ сильно положительны.

Теорема 1. Для любых $F_{1}, F_{2}$ из $B V[0, l]$ задача (1)-(6) однозначно разрешима (при $\left.f_{1}=F_{1}^{\prime} u f_{2}=F_{2}^{\prime}\right)$.

Теорема 2. Пусть $F_{1}$ и $F_{2}$ - первообразные функиий $f_{1} u f_{2}$. Тогда для любых неубъвающ, их $F_{1}, F_{2}$ при наличии хотя бъ одной точки роста $F_{1}$ или $F_{2}$ решение $u(x)=\left\{u_{1}(x)\right.$, $\left.u_{2}(x)\right\}$ строго полохительно в $(0, l)$, т.е. $u_{1}(x)>0 u u_{2}(x)>0$ на $(0, l)$. Более того, для любих двух "неотрицательных" пар $\left\{f_{1}, f_{2}\right\}$ соответствующие им решения $u(x)=$ $\left\{u_{1}(x), u_{2}(x)\right\}$ и $v(x)=\left\{v_{1}(x), v_{2}(x)\right\}$ соизмеримы по конусу неотрицательных функций $в$ $C[0, l] \times C[0, l]$, m.e.

$$
\sup _{x, s} \frac{u_{i}(x)}{v_{i}(x)} \frac{v_{i}(s)}{u_{i}(s)}<\infty \quad n p u \quad i=1,2 .
$$

Последнее свойство означает усиленную положительную обратимость задачи (1)-(6). Более сильно это свойство можно описать так. Пусть $K$ - конус неотрицательных функций в пространстве $C[0, l] \times C[0, l]$. Пусть $A$ - обратный к задаче (1)-(6) оператор. Стандартным способом проверяется, что он имеет интегральный вид

$$
\left(A F^{\prime}\right)(x)=\int_{0}^{l} G(x, s) d F(s)
$$

где $G(x, s)$ - двумерная матрица-функция Грина. Определяемый этой функцией интегральный оператор действует в $E=C[0, l] \times C[0, l]$ и сильно положителен на конусе $K$. 
2. Рассмотрим вместо (1), (2) уравнения

$$
\left(p_{1} u_{1}^{\prime \prime}\right)^{\prime \prime}=\lambda M_{1}^{\prime} u_{1}, \quad-\left(p_{2} u_{2}^{\prime}\right)^{\prime}=\lambda M_{2}^{\prime} u_{2}
$$

где $M_{1}, M_{2}$ - неубывающие функции, определяющие распределение масс соответственно на стержне и струне. Следующая теорема устанавливается на основе описанного свойства оператора (8).

Tеорема 3. Пусть одна из функиий $M_{1}(x), M_{2}(x)$ имеет хотя бы одну точку роста (т.е. отлична от константы). Тогда минимальное по модулю собственное значение $\lambda_{0}$ задачи (8) при условиях (3)-(6) является строго полохительным и простым (корневое пространство одномерно), любая другая точка $\lambda$ спектра удовлетворяет неравенству $|\lambda|>\lambda_{0}$. Соответствующая $\lambda_{0}$ собственная функиия $u^{\circ}(x)$ имеет обе строго полохительные $($ на $(0, l))$ компоненты.

Задача (1)-(6) оказьвается самосопряженной в естественном смысле, ее функция-матрица Грина - симметричным положительньп ядром. Поэтому весь спектр задачи (8), (3)-(6) состоит из вещественных положительных чисел. По всей видимости, все они простые, а соответствующие им собственные функции имеют (как в классической теории Штурма-Лиувилля) количество перемен знака, совпадающее с номером соответствующего собственного значения (в естественной иерархии). Однако даже набор слов "число перемен знака", очевидный для скалярных функций, допускает самые разные толкования для вектор-функций, и потому описание осцилляционных свойств собственных функций в рассматриваемом случае есть задача будущего.

3. Функция-матрица Грина $G(x, s)$ задачи (1)-(6) допускает стандартное задание через фундаментальную систему решений однородного "уравнения"

$$
\left(p_{1} u_{1}^{\prime \prime}\right)^{\prime \prime}=0, \quad\left(p_{2} u_{2}^{\prime}\right)^{\prime}=0, \quad x \neq \xi
$$

где, аналогично взглядам теории уравнений на графах (см. библ. в [2]) условия (3)-(5) удобно отнести к определению решения и, более того, к толкованию обобщенного уравнения (9) в точке $x=\xi$. Функция $G(x, s)$ оказьвается непрерывной на $[0, l] \times[0, l]$ и строго положительна (по каждой координате) внутри этого квадрата. Следует отметить, что даже непрерьвность здесь - весьма непросто проверяемое свойство. Трудности сосредоточены в окрестности прямой $s=\xi$. Подобные трудности нетривиальны даже для скалярных задач с внутренними особенностями.

Для вектор- функции символ “\$” означает у нас синхронное выполнение по обеим компонентам аналогичного скалярного неравенства.

Tеорема 4. Существует строго положительная функция $\varphi(x) \quad\left(=\left\{\varphi_{1}(x), \varphi_{2}(x)\right\}\right)$ такая, что $\varphi(x) G(\tau, s) \leqslant G(x, s)$ при всех $x, \tau, s$ из $[0, l]$.

Следствием этого факта для оператора (7) является неравенство $\varphi(x) \max _{\tau}(A z)(\tau) \leqslant(A z)(x)$ для любой $z(x) \geqslant 0$, где неравенства (и максимум) понимаются в синхронно-двухкомпонентном плане. Отсюда следует аналог классического свойства Харнака: для любого нетривиального решения $и(x) \quad\left(=\left\{u_{1}(x), u_{2}(x)\right\}\right)$ неравенств

$$
\left(p_{1} u_{1}^{\prime \prime}\right)^{\prime \prime} \geqslant 0, \quad-\left(p_{2} u_{2}^{\prime}\right)^{\prime} \geqslant 0
$$

при условиях (3)-(6) имеет место $\varphi(x) \max _{\tau} u(\tau) \leqslant u(x)$. 


\section{СПИСОК ЦИТИРОВАННОЙ ЛИТЕРАТУРЫ}

1. Покорный Ю. В., Мустафокулов Р. // Дифференц. уравнения. 1977. Т. 33. № 10. С. 1358-1365. 2. Покорный Ю. В. // Дифференц. уравнения. 2001. Т. 37. № 5. С. 661-671.

(Ю. В. Покорный) Воронежский государственный университет

Поступило

(Т.В. Белоглазова) Воронежский государственный университет

(Е. В. Дикарева) Воронежский институт МВД РФ

(Т.В. Перловская) Воронежская лесотехническая академия

E-mail: (10. В. Покорньй) pokorny@kma.vsu.ru,

(Т. В. Бөлоглазова) sprite2000@mail.ru 